duced by the dynamic-Stark splitting of level a by the dye laser field) develops a narrow central feature as the magnetic field is increased, as predicted by Cohen-Tannoudji et al. The work has novel implications for Doppler-free superradiance, the observation of Doppler-free coherent transients, and predicts striking forward-backward asymmetries in spectral profiles of great interest in intracavity ring systems.

\title{
Interferon and double-stranded RNA
}

from John Morser and Derek Burke

THE pas de deux between interferon and double-stranded RNA (dsRNA) continues (see News and Views 273,$97 ; 1978$ ). It has been known for many years that treatment of cells with double-stranded RNA induces interferon. It now seems likely that viruses also induce interferon by virtue of their ability to make double-stranded RNA, as shown by the elegant study of Marcus and Sekellick (Nature 266, 815; 1977), although additional factors might also be involved (see Kowal \& Youngner Virology 90, 90; 1978). When either double-stranded RNAs or viruses are used as inducers, the interferon yield can often be increased by pretreatment of cells with interferon ('priming'). Priming is specific for interferon and it may often turn a non-inducible system into an interferonproducing system. Some cells, notably mouse L cells, also show a pronounced cytotoxicity when treated with interferon and double-stranded RNA (Stewart et al. Proc. natn. Acad. Sci. U.S.A. 69, 1851; 1972). Since such cells also produce interferon, interferon is being synthesised by cells undergoing pronounced cytotoxic damage. Finally, when interferon-treated cells are exposed to double-stranded RNA, translation is blocked, and a series of recent papers (Kerr \& Brown Proc. natn. Acad. Sci. U.S.A. 75, 256; 1978; Ball \& White Proc. natn. Acad. Sci. U.S.A. 75, 1167; 1978; Lewis et al. Eur. J. Biochem. 86, 497; 1978; Schmidt et al. FEBS Lett. 95, 257; 1978; Farrell et al. Proc. natn. Acad. Sci. U.S.A. 75, 5893; 1978) shows that at least two separate mechanisms are involved. One involves the activation of a protein kinase that phosphorylates, and consequently inactivates the initiation factor eIF-2, and the other involves the formation of pppA2'p5'A2'p5'A ('two-five A') which in turn activates a constitutive inactive endonuclease that degrades mRNA (see diagram).

These effects of interferon require the presence of chromosome 21 in human cells, probably to make the interferon receptor on the cell surface. Both pathways that lead to inhibition of protein synthesis require ATP: the pathway resulting in inhibition of peptide chain initiation for the activation of the protein kinase system, and the one resulting in mRNA degradation to synthesise two-five A by the interferoninduced oligo-isoadenylate synthetase The scheme reveals a paradox: cells in which protein synthesis has been impaired by two separate mechanisms are also the cells in which interferon mRNA is being actively translated. How can a new protein be formed in such cells? Are any of the intermediates involved in the induction of the interferon gene? The enzyme that synthesises two-five A is possibly one candidate, since it complexes sufficiently well with double-stranded RNA for that to be used as a purification method (Hovanessian et al. Nature 268, 537; 1977), and seems to be present in small amounts in cells which have not yet been exposed to interferon.

One generalisation can be made, however. Interferon sensitises the cell to double-stranded RNA-so that a series of processes which previously occurred either not at all, or at low levels, is now activated. Perhaps this is the control function of interferon-

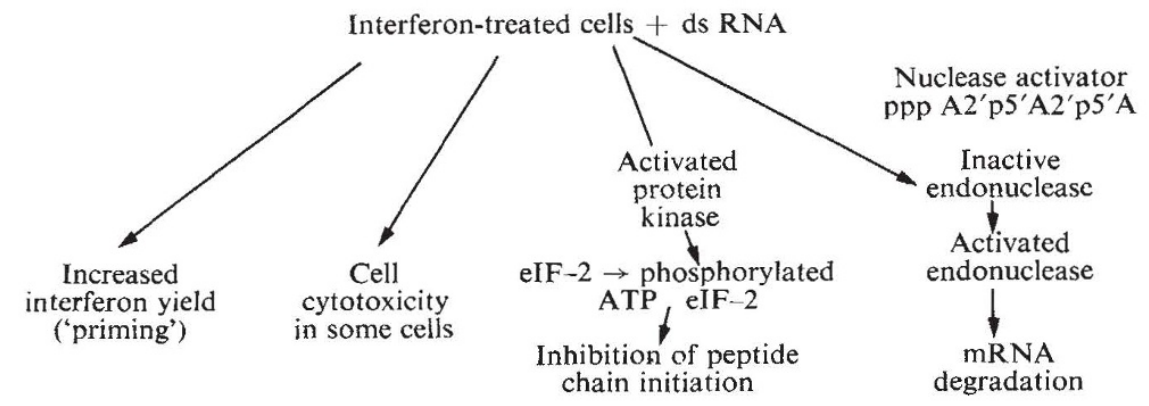

to tell the cells to respond to dsRNA - either added as a synthetic dsR NA (poly ( $\mathrm{rI}$ ) poly $(\mathrm{rC})$ ) or formed as a byproduct of virus multiplication.

It is well known that reticulocyte lysates are exquisitely sensitive to the effect of dsRNA, much more sensitive than are cell-free systems made from normal cells and as sensitive as cellfree systems made from interferontreated cells. Hunt (News and Views $o p$. cit.) has suggested that this is because erythrocyte differentiation depends on exposure to interferon at some stage. Certainly interferon antagonises the effect of colony-stimulating factor, inhibits the growth of haemopoetic colony-forming cells and interferes with the normal differentiation of granulocyte-macrophage precursors (McNeil \& Fleming in The Interferon System: a Current Review to 1978, Texas Reports on Biology \& Medicine (eds Baron \& Dianzani) 343; 1978). Interferon also inhibits the expression of erythroid differentiation in Friend leukaemia cells treated with dimethylsulphoxide (Rossi et al. ibid page 420).

If interferon is involved in any way in differentiation, is this the reason why the interferon system is an inducible rather than a constitutive system and why it is completely uninducible in mouse teratocarcinoma stem cells (Burke et al. Cell 13, 243; 1978)? Perhaps dsRNA is a pleiotropic effector, but only active in interferon-treated cells? We do not yet know the answers to these questions, but perhaps we should look harder for double-stranded RNA elsewhere.

J. Morser is a lecturer and D. C. Burke is Professor in the Department of Biological Sciences, University of Warwick.

\section{Radioactive waste management}

from $S$. Weisenburger

THIS interdisciplinary meeting*, covering a broad range of topics, from the processing, solidification, and characterisation to the isolation of radioactive wastes arising from the nuclear fuel cycle was devoted especially to scientific research aspects of radioactive waste management. These aspects have often been relegated to the sidelines in international gatherings chiefly concerned with systems management.

A general review on the management of nuclear waste by Rustum Roy

*An International Symposium on 'Science underlying waste management" was held in Boston on 28 November-1 December, 1978 and sponsored by the Materials Research Society. The proceedings will be available in Spring, 1979. 